\title{
Eine Aufgabe mit Schlüsselfunktion
}

Erfahrungsbericht Im Rahmen ihres Studiums Master of Science in Nursing an der Zürcher Hochschule für Angewandte Wissenschaften in Winterthur absolvierte Cornelia Stricker ein Workshadowing im Pflegedienst des Universitätskinderspitals Zürich. Innerhalb von zwei Wochen erhielt sie einen direkten Einblick in die Pflegepraxis der Pflegeexpertinnen APN Kardiologie, APN Onkologie und APN Kontinenz und über ihre Verankerung in der klinischen Praxis. Hier beschreibt sie die vielfältigen Aufgaben pädiatrischer Pflegeexperten APN in der klinischen Praxis und zeigt Möglichkeiten des Umgangs auf.

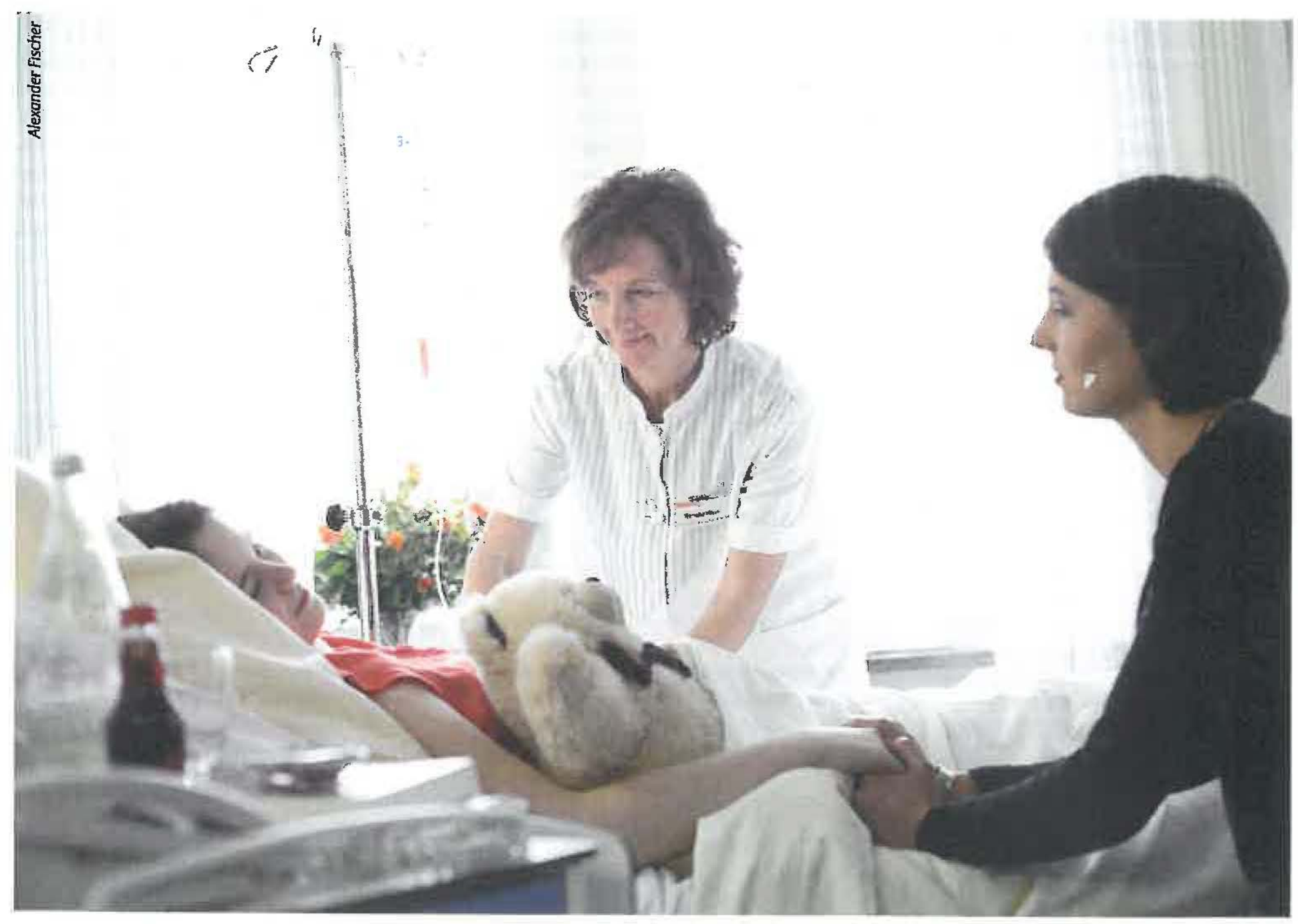


- Cornelia Stricker, Anna-Barbara Schlüer, Daniela Händler-Schuster -

Der medizinische Fortschritt und die dadurch verbesserten Behandlungsmöglichkeiten in der Pädiatrie führten in den letzten Jahrzehnten dazu, dass die Überlebenschance von Kindern und Jugendlichen mit schweren angeborenen oder erworbenen Krankheiten sowie nach Unfällen zunahm. ${ }^{1,2}$ Immer mehr Kinder und Jugendliche können nach Abschluss der Akutbehandlung ein beschwerdefreies Leben führen. Manche sind jedoch während mehrerer Monate, Jahre oder sogar ihr Leben lang von Einschränkungen in ihrem Alltag betroffen. In der Schweiz trifft dies auf 8,7\% der Kinder im Alter bis zu zehn Jahren zu. ${ }^{2}$ Ursache hierfür kann das Überstehen einer schweren Krankheit oder eines Unfalls sein, eine angeborene Beeinträchtigung oder ein chronischer Verlauf. ${ }^{1}$ Die Literatur beschreibt unter anderem folgende mögliche Einschränkungen im Alltag: ${ }^{1,2}$

- Tägliche, exakte Medikamenteneinnahme nach einer Organtransplantation

- Mehrfach tägliches Inhalieren zur Sekretolyse bei zystischer Fibrose

- Eingeschränkte Mobilität bei juveniler rheumatoider Arthritis
- Verzicht auf gefährliche (Freizeit-)Aktivitäten aufgrund einer Hämophilie

Eine weitere Veränderung im Gesundheitswesen ist die zunehmende Versorgungsverlagerung vom Krankenhaus weg in den ambulanten Bereich. Durch diesen Wandel ändern sich die Bedürfnisse der pädiatrischen Patienten und ihrer Familien. Sie übernehmen beispielsweise vermehrt Aufgaben, die bislang zum Tätigkeitsfeld des Pflegepersonals in der Klinik gehörten. ${ }^{1,2}$

Um trotz der zunehmenden komplexen Patientensituationen und der verkürzten Hospitalisationszeiten eine kontinuierliche Patientenversorgung gewährleisten zu können, sind neue Versorgungsmodelle im Gesundheitswesen erforderlich.3,4

Eine mögliche Antwort auf die hoch spezialisierte Medizin ist der Bedarf nach einer ebenso hoch spezialisierten Pflege. Eine Schlüsselrolle hat dabei die Pflegeexpertin Advanced Practice Nurse (APN) inne, die über erweiterte und vertiefte Fachkenntnisse verfügt. ${ }^{5}$ Ihre zentrale Kompetenz ist die direkte klinische Tätigkeit, ergänzt durch folgende sechs Kernkompetenzen: Coaching und Führung, Beratung und Konsultation, Forschungsfertigkeiten, klinisches und berufspolitisches Leadership, Zusammenarbeit und Teamfähigkeit sowie Fähigkeiten zur ethischen Entscheidungsfindung. ${ }^{6}$ Durch ihren Einsatz kann eine qualitativ hochwertige pflegerische Versorgung im Sinne einer Advanced Nursing Practice (ANP) sichergestellt werden. ${ }^{3}$

\section{APN am Kinderspital Zürich}

Das Kinderspital Zürich ist in der Schweiz das größte universitäre Zentrum für die Versorgung, Bildung und Forschung in der Kinder- und Jugendmedizin. Im Bereich der Pflege zeichnet sich das Kinderspital heute vor allem darin aus, dass es eine Vorreiterfunktion eingenommen hat bezüglich der Implementierung und Weiterentwicklung der Pflegeexperten APN in der Schweiz. ${ }^{7}$ Unter ANP wird heute eine vertiefte und erweiterte klinische Praxis auf den Grundlagen fundierter wissenschaftlicher Erkenntnisse verstanden. ${ }^{3}$

Der Mangel an universitär ausgebildeten Pflegespezialisten in der Schweiz war anfangs eine große Herausforderung. Um diesem Umstand entgegenzuwirken, entstanden sogenannte ANP-Teams. Bei diesem Ansatz werden ganze Teams von Pflegenden mit verschiedenen Ausbildungen

\section{Fallbeispiel}

Ein Tag mit viel Eigenverantwortung

Es ist 8 Uhı morgens, als ich Lena treffe, Pflegeexpertin APN Kontinenz. Ein erster Blick in ihre Mallbox gibt bereits Hinweise auf einen arbeitsreichen Tag. Wir verschaffen uns jedoch zuerst einen Uberblick uber die urologischen Patienten aứ der chirurgischen Station Gleich beginnt die Visite, geführt durch den urologischen Oberarzt und Assistenzarzt. Die Pflege ist durch die zustandige diplomierte Pflegefachperson und Lena vertreten Hierbei unterstutzt Lena einerseits Mitarbeitende des Pflegedienstes mit geringer Erfahrung auf dem Fachgebiet der Urologie und anderer seits das Pflegeteam in komplexen Patientensituationen. Wahrend der Visite klingelt mehrfach Lenas Telefon. Als cile Visite beendet ist, begeben wir uns auf die Notfallstation, da das Pflegepersonal uns um Unterstutzung bat Ein zwölfjährıger Junge mit einem Nabelstoma, dem es seit dem Vorabend nicht mehr gelingt sich selbst zu katheterisieren, ist eingetroffen Gemeinsam mit Lena versucht er im Liegen, Sitzen und Stehen den Katheter einzulegen Sie gibt ihm Tipps, wie etwa den Bauch oberhalb des Nabels etwas nach innen zu drucken und die Bauchmuskulatur zu entspannen. Nach mehreren Versuchen und mit einem etwas dunneren Katheter gelingt schließlich die Kathetereinlage Zuruck auf der Station werden wir bereits von einer Familie zum Beratungsgespräch erwartet. Das etwa sechsjährige Mädchen ist aufgrund einer angeborenen Nervenschadigung stuhl- und urinınkontinent. Selbststandig erfasst Lena eine umfassende Anamnese, um sich einen Überblick uber die aktıelle Problematik zu verschaffen. Anschließend führt sie eine körperliche Untersuchung durch und formuliert einen Therapievorschlag Ihre Beobachtungen und Untersuchungsergebnisse schildert sie telefonisch der zustandigen Arztin und stellt ihren Therapievorschlag vor. Die Arztin bestatigte diesen und stellt das Rezept fur die notwendigen Medikamente aus Den Gesprachsabschluss bilden die Besprechung des Therapieplans mit der Familie und die Klärung letzter Fragen. Mittlerweile ist es bereits $1240 \mathrm{Uhr}$, als wir im Personalrestaurant unsere Pause antreten. Viel Zeit bleibt abes nicht Um 13 Uhr beginnt die urologische Sprechstunde Deren Besonderheit besteht darın, dass sle gemeinsam von der urologischen Oberarztin und der Pflegeexpertın APN Kontinenz durchgeführt wird. Als die Sprechstunden beendet sind und wir uns ins Buro zuruckziehen, ist es bereits $1520 \mathrm{Uhr}$ Jetzt ist endlich Zeit, um Ruckrufe zu tat.ıgen und Mails zu beantworten. Mehrere Eltern haben sich bei Lena per Mall gemeldet, da irı der Betreuung ihrer kranken Kinder zu Hause Fragen oder Unsicherheiten auftraten. Die Bandbreite der Anfragen geht von administrativen Belangen bezuglich eines Kontrolltermins bis hin zu pflegerischen Fragen, wie etwa dem korrekten Anwenden der Abfuhrmaßnahmen bei Obstipation. Gegen 17 Uhr ist schließlich der wohlverdiente Felerabend erreicht. 


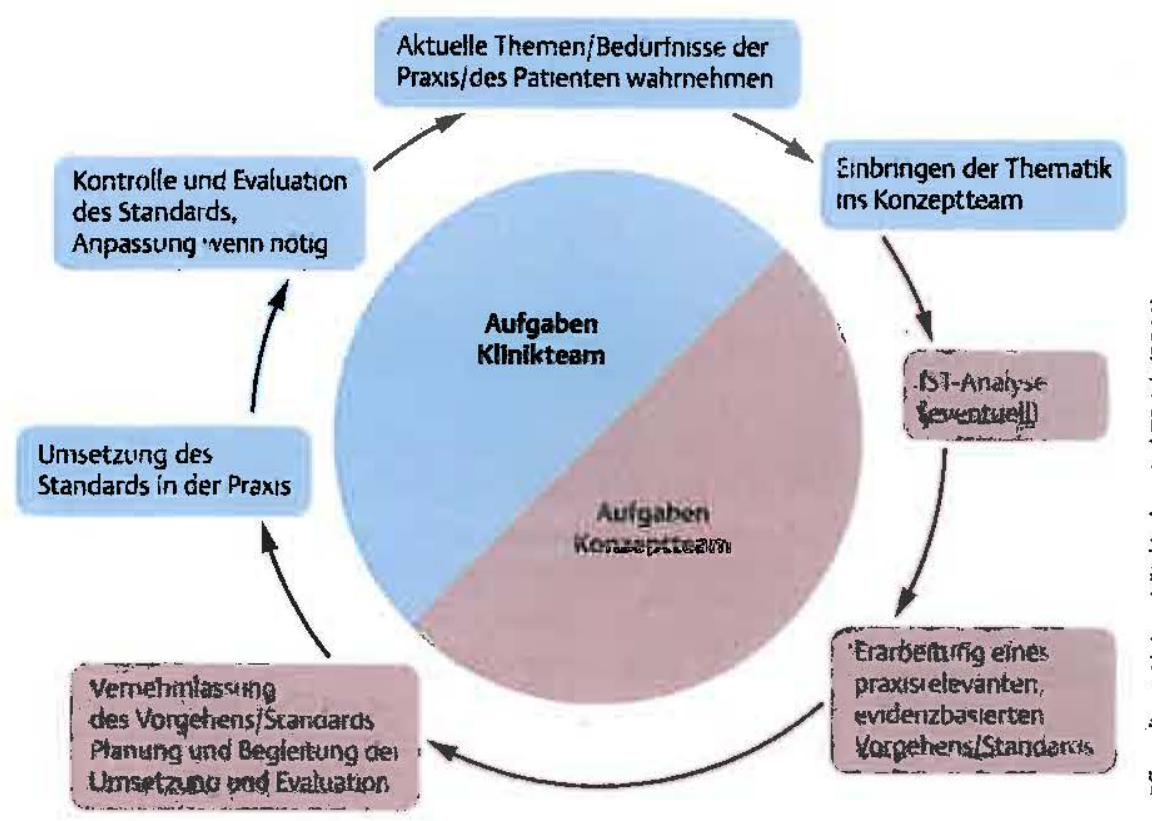

Abb. 1 Aufgabenverteilung innerhalb der ANP-Teams.

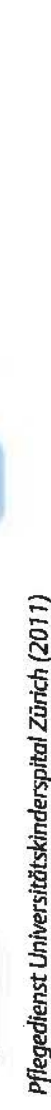

dazu befähigt, unter Leitung der Pflegeexpertin APN eine Pflegepraxis im Sinne von ANP anzubieten. Dies ermöglicht die gezielte Förderung der Pflegepraxis trotz des bestehenden Spannungsfelds zwischen hoch komplexen Situationen und oft begrenzten Ressourcen. ${ }^{5}$ Demgegenüber steht die Pflegeexpertin APN als Einzelrolle. Wird ein Kind oder Jugendlicher hauptsächlich innerhalb eines Fachbereichs versorgt, erfolgt die pflegerische Begieitung und Betreuung durch eine Pfiegeexpertin APN in der Einzelrolle, wie etwa bei Kindern mit einer Hämophilie. Erfolgt die Behandlung jedoch über mehrere Fachbereiche und Stationen, wie bei onkologischen oder kardiologischen Patienten, wird der Teamansatz favorisiert. ${ }^{7}$

\section{ANP-Teams - \\ Mitglieder und Aufgaben}

Aktuell bietet das Kinderspital Zürich rund zehn ANP-Spezialisierungen an, wie beispielsweise im Fachgebiet der Nephrologie, Hämatologie oder in der pädiatrischen Palliative Care. ${ }^{8}$ Jedes ANP-Team wird in ein Konzept- und ein Klinikteam aufgeteilt $(\rightarrow$ Abb. 1). Die Mitglieder des Konzeptteams, unter der Leitung der Pflegeexpertin APN, verfügen über eine höhere pflegerische Ausbildung im Sinne eines Bachelors oder Masters of Science oder eines Masters of Advanced Studies. Sie erarbei- ten Standards und Konzepte unter Einbezug praxisrelevanten und evidenzbasierten Fachwissens. ${ }^{5}$ Das Klinikteam besteht aus erfahrenen Mitarbeitenden verschiedener Fachbereiche, die über eine hohe Akzeptanz in der klinischen Praxis verfügen und andere motivieren und befähigen können. Ihr Schwerpunkt ist die Implementierung von Neuerungen in die Praxis. Eine weitere Aufgabe der ANP-Teams ist die umfassende Beratung und Schulung von Kindern, Jugendlichen und ihrer Familien bezüglich Erkrankung, Symptommanagement und Alltagsbewältigung. 5

Während des Workshadowings erhielt ich in verschiedene ANP-Teams Einblicke. Wie ein Arbeitstag der Pflegeexpertin APN Kontinenz aussieht veranschaulicht das $\rightarrow$ Fallbeispiel.

\section{Herausforderungen im klinischen Alltag}

Vergleichend mit der Literatur können verschiedene Herausforderungen beschrieben werden, die im klinischen Alltag pädiatrischer Pflegeexperten APN auftreten, wie das oben beschriebene Beispiel bereits aufzeigt. Diese können in Bezug auf das erweiterte, vertiefte Fachwissen, aber auch bezüglich der Ressourcen, die häufig personell und zeitlich eher knapp sind, beschrieben werden. Zudem ist die Zusammenarbeit zwischen Pflegeexperten APN

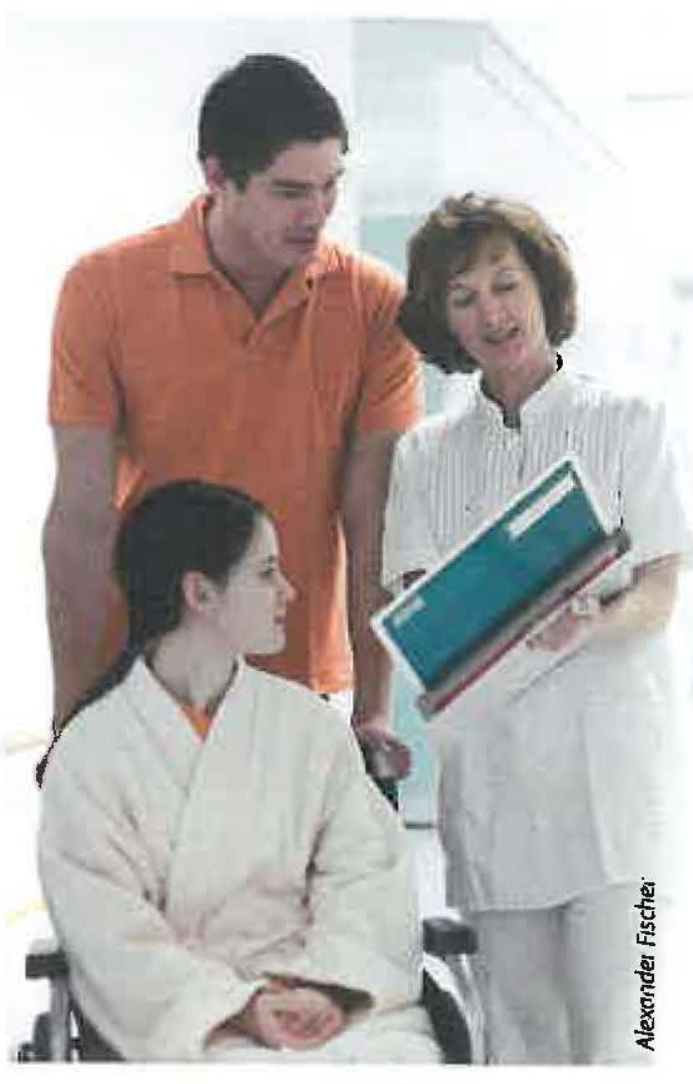

Beratungsgespräche mit Patienten und Angehörigen gehören ebenso dazu wie...

und dem ärztlichen Dienst immer wieder ein Thema.

\section{Erweitertes, vertieftes Fachwissen}

Eine Herausforderung liegt in der sicheren Ausübung der eingangs beschriebenen vielfältigen APN-Tätigkeiten. Manche Pflegeexperten APN berichten über einen Mangel an klinischer Fachkompetenz, Unsicherheiten in der selbstständigen Fallführung oder bei der Erhebung einer umfassenden Anamnese und de-: Durchführung des klinischen Assessments. Dies wird auf die zu seltene Anwendung dieser Tätigkeiten nach dem Studium zurückgeführt. ${ }^{4,5}$ Doch nur mit erweitertem und vertieftem Wissen ist es möglich, fachlich korrekt die eigene Position gegenüber anderen Berufsgruppen zu vertreten. 5

\section{Knappe personelle} und zeitliche Ressourcen

Das Angebot der Pflegeexperten APN deckt bisher nicht alle Bedürfnisse der Betroffenen, ihrer Familien und der Fachpersonen ab. Dies zeigt sich beispielsweise in der verminderten Verfügbarkeit ihrer Person. Daher ist unter anderem die Ausweitung des Angebots notwendig, einerseits für Betroffene und ihre Familien, andererseits für Teachings und Coachings von Fachpersonen. ${ }^{3}$ Um die Bedeutung der Pflegeexperten APN in der Praxis zu festigen, ist es 


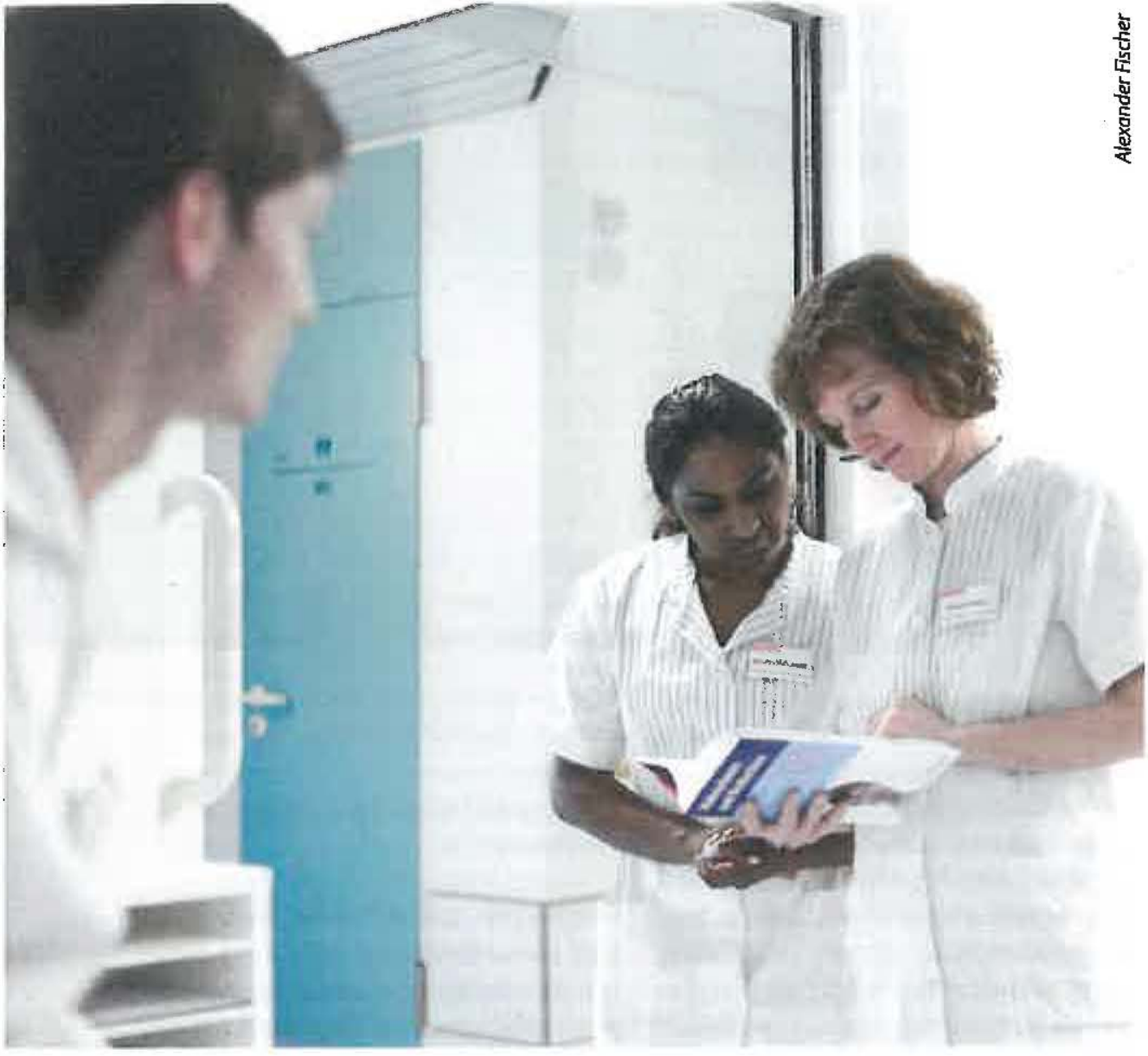

... das Besprechen von therapeutischen Maßnahmen mit betreuenden Kolleginnen ...

notwendig, dass wissenschaftliche Evaluationen ihrer Tätigkeiten und deren Auswirkungen erfolgen. Doch im Spannungsfeld der knappen zeitlichen, personellen wie auch finanziellen Ressourcen ist dies eine schwierige Aufgabe. Daher erfolgte in den vergangenen Jahren nur selten eine Evaluation der APN-Tätigkeiten. ${ }^{5}$

Interprofessionelle Zusammenarbeit In der Praxis treten teilweise Doppelspurigkeiten zwischen Tätigkeiten des ärztlichen Dienstes und denen der ANP-Teams auf. Um diesen Umstand zu vermeiden, ist eine gezielte Kommunikation seitens Pflege und Arzt erforderlich. ${ }^{3}$ Damit eine konstruktive Zusammenarbeit möglich ist, bedarf es einer klaren Rollendefinition der Pflegeexperten APN. ${ }^{3}$ Ärzte, die diese Rolle bereits aus dem Ausland kennen, haben exkannt, dass es sich bei den Tätigkeitsfeldern der Pflegeexperten APN nicht um eine Übernahme ärztlicher Arbeiten geht.
Sprache kamen, die auch nach 15 Jahren Praxiserfahrung im Kinderspital Zürich bestehen. Teilweise decken sich diese mit der Literatur.

\section{Erweitertes, vertieftes Fachwissen}

Die Arbeit in den ANP-Teams erlebte ich als professionell und fachlich fundiert. Dies ist im beschriebenen Beispiel an der selbstständigen Erhebung der Anamnese, dem gezielten körperlichen Assessment und dem daraus resultierendem Therapievorschlag der Pflegeexpertin APN zu erkennen. Durch den Einblick in die verschiedenen ANP-Teams erkannte ich, dass trotz eines spitalinternen ANP-Konzepts Unterschiede im klinischen Alttag bestehen und Freiräume vorhanden sind. Dies bestärkt mich darin, meine eigene Vision der Berufsrolle Pflegeexpertin APN auf einer Intensivstation weiterzuverfolgen, proaktiv die eigene Berufsrolle mitzugestalten und persönliche Ideen und Vorstellung einflieBen zu lassen.

\section{Knappe personelle} und zeitliche Ressourcen

Als eine große Hürde erkannte ich während meines Workshadowings vor allem die personellen und zeitlichen Ressourcen. Den vielfältigen Ansprüchen seitens Patienten, Familien und Fachpersonen gerecht zu werden bei gleichzeitiger Ausübung aller Kernkompetenren der Pflegeexperten APN - das ist im Spannungsfeld der knappen Ressourcen schwierig. Dies zeigte sich daran, dass die Forschungsfertigkeit im klinischen Alltag oft zu kurz kommt und Evaluationen der APN-Tätigkeiten nur selten stattfinden. te ausstellen dürfen. Andererseits ist die Abrechnung der pflegerischen Leistungen und somit die Finanzierung dieser Berufsrolle noch nicht umfassend möglich. Hierfür fehlen derzeit die Gesetzesgrundlagen in der Schweiz. ${ }^{4}$

\section{Persönlicher Rückblick auf das Workshadowing}

Das Workshadowing ermöglichte mir viele interessante und spannende Einblicke in die verschiedenen ANP-Teams. Ich erkannte, dass auch nach vielen Jahren Praxiserfahrung neue Aufgaben auftauchen. In den Gesprächen mit den ANP-Teams stellte ich fest, dass oftmals ähnliche Themen zur
Interprofessionelle Zusammenarbeit schrieben erlebte ich die Zusammenarbeil mit dem ärztlichen Dienst. Jeder wusste was innerhalb der Behandlung seine Auf. gaben sind, und ein regelmäßiger Aus. tausch fand statt. Im Alltag der Pflegeex. pertin APN Kontinenz zeigt sich dies in de: morgendlichen Visite, der gemeinsamer Sprechstunde und im Therapievorschlas seitens der Pflege. Im ANP-Team Kardio. logie erlebte ich im Rahmen des tägliches interdisziplinären Morgenrapports einı andere Art der Zusammenarbeit. Unte Komplett anders als in der Literatur be- 


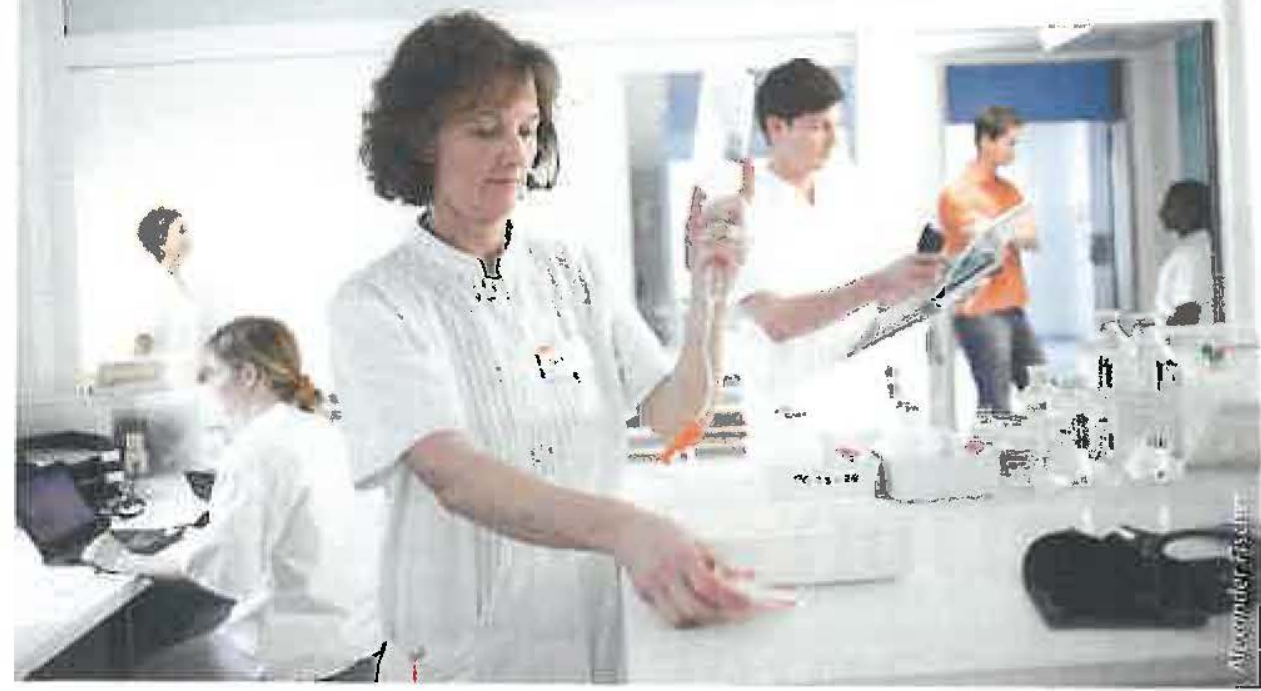

... und auch die Medikamentenversorgung ist Teil der Aufgaben einer Pflegeexpertin APN.

ärztlicher Leitung wurden die kardiologischen Patienten vorgestellt, aktuelle Befunde gemeinsam betrachtet und die Prozederen besprochen. Dadurch sind alle an der Behandlung beteiligten Personen auf dem gleichen Wissensstand - die Grundlage einer konstruktiven Zusammenarbeit.

Eine Stärke der APN-Arbeit, die mich besonders beeindruckt hat, ist die Kontinuität in der Betreuung und Begleitung kranker Kinder und ihrer Familien. Die Pflegeexpertin APN ist zu jeder Zeit die erste Ansprechperson für Betroffene - und zwar vor einem Spitalaufenthalt, währenddessen und danach. Sie organisiert beispielsweise eine spitalnahe Unterkunftsmöglichkeit für Eltern, erteilt Auskunft über den Stand der laufenden Operation und gibt im Rahmen von Schulungen und Anleitungen ihr Wissen und Können weiter. Ihre tägliche Präsenz auf den Stationen erachte ich hierbei als sehr förderlichen Faktor, um mit allen Beteilig-

ten einen regelmäßigen Austausch zu pflegen.

\section{Literatur}

1 van der Lee JH, Mokkink LB, Grootenhuis MA Heymans HS, Offringa M. Definitions and measurement of chronic health conditions in childhood - a systematic Revlew. The Journal of the American Medical Association 2007; 297(24): 2741-2751

2 Schweizerisches Gesundheitsobservatorium. Gesundheit in der Schweiz - Fokus chronische Erkrankungen - Nationaler Gesundheitsbericht 2015. Online unter www.obsan.admin.ch/de publikationen, letzter Zugriff 26.102016

3 Waldboth V, Schlūer AB, Müller-Staub M. Evaluation pädiatrischer Advanced Practice Nurses: Qualităt und Nutzen für die Schweiz? Pflege 2013; 26(6): 421-430

4 Ullmann P, Schwendimann R, Keinath E, Eder K, Henry M, Thissen K, Schönthaler A, Mauthner O, Freyer S, Fierz K, Gantschnig G, Stoll H, Nicca $D$, Lehwaldt D, Ullmann B. Visionen und Realitäten in der Entwicklung von APN \& ANP. Advanced Practice Nurses MAGAZIN 2015; 8-23

5 Ullmann-Bremi A, Schlüer AB, Finkbeiner G, Huber Y. "Wie ein ANP-Team laufen lernt" - Herausforderungen und Chancen von ANP-Teams am Universitätskinderspital Zürich. Pflege 2011; 24(1): 21-28

6 Hamric AB, Hanson CM, Tracy MF, O'Grady ET. Advanced Practice Nursing - An Integrative Approach. 5th ed. St. Louis, Missouri: Elsevier; 2014

7 Pflegedienst Universitätskinderspital Zürich. Pflegesicht - Sonderausgabe 10 Jahre ANP. Universitätskinderspital Zúrich 2011. Online unter https://www.kispi.uzh.ch/de/UeberUns/
spitalorganisation/pflegedienst/Seiten/default. aspx, letzter Zugriff 07.10.2016

8 Pflegedienst Universitătskinderspital Zürich. Advanced Nursing Practice (ANP). Universitätskinderspital Zürich 2016. Online unter https:// www.kispi.uzh.ch/de/Gesundheitsberufe/ fachinformationen_pflege/anp/Seiten/default. aspx, letzter Zugriff 12.11.2016

\section{Autorinnen}

\section{Cornelia Stricker}

MScN. Als Fachexpertin Pflege auf der Intensivstation im Spital Zollikerberg tätig. Mitglied in der Interessensgruppe Praxisentwicklung Intensivpflege in der schweizerischen Gesellschaft für Intensivmedizin (SGI).

E-Mail: conny_stricker@hotmail.com

\section{Dr. Anna-Barbara Schliler}

PhD, MScN, RN, Pflegeexpertin APN Wund-, Haut- und Stomapflege und Leltung klinische Pflegewissenschaft am Kinderspital Zürich.

\section{Prof. Mag. Dr. rer. medic.}

\section{Daniela Hăndler-Schuster}

Dipl.-Berufspäd. FH. Professorin für gemeindenahe integrierte Pflege. Forschungsschwerpunkte sind u. a. das Leben mit Beeinträchtigungen in unterschiedlichen Lebensphasen (z. B. psychische oder kognitive Beeinträchtigungen; Alltagsbewältigung von Kindem und Jugendlichen mit Hörbehinderung), die interprofessionelle Zusammenarbeit (z. B. in der Gerontechnologie) sowie die Professionalisierung der Pflege.

\section{Danksagung}

Ein besonderer Dank geht an die drei ANP-Teams der Kardiologie, Onkologie und Kontinenz für die spannenden Einblicke.

\section{Bibllografie}

DOI 10.1055/s-0043-105404

JuKiP 2017; 3: 105-109

(O) Georg Thieme Verlag KG

Stuttgart - New York - ISSN 1439-2569

Fandt

Während des Workshadowings wurde mir die Bedeutung der interprofessionellen Zusammenarbeit eindrücklich vor Augen geführt. Egal in welchem Bereich: Die Pflegeexpertin APN ist stets ein Teil des Behandlungsteams. Um eine optimale Betreuung und Begleitung zu ermoglichen, ist eine gezielte Kommunikation zwischen den einzelnen Diensten notwendig, um Wissen zu teilen und Ressourcen optimal zu nutzen. Weiter zeigte mir dieser Praxiseinblick auf, dass das Lernen nach dem Masterstudium nicht aufhört. Um die gelernten APN-Fähigkeiten beizubehalten, sind die direkte klinische Praxis und das regelmaßıge Anwenden dieser Tätigkeiten essenziell. Hinzu kommt, dass die Praxisentwicklung ein kontinuierlicher (Lern-)Prozess ist, in dem auch das Pflegemanagement von besonderer Bedeutung ist. Im Kinderspital Zürich spürte ich rasch, dass die ganze Institution hinter dem Konzept ANP steht - vom ärztlichen Dienst über das Pflegemanagement und die Pflegeentwicklung bis hin zu den einzelnen Fachpersonen der jeweiligen Stationen. Eine enge Zusammenarbeit aller Dienste ist zwingend erforderlich, um die zahlreichen Herausforderungen im Alltag mit kranken Kindern und ihren Eltern erfolgreich zu meistern und eine hoch spezialisierte, evidenzbasierte Pflegepraxis anbieten zu konnen. 